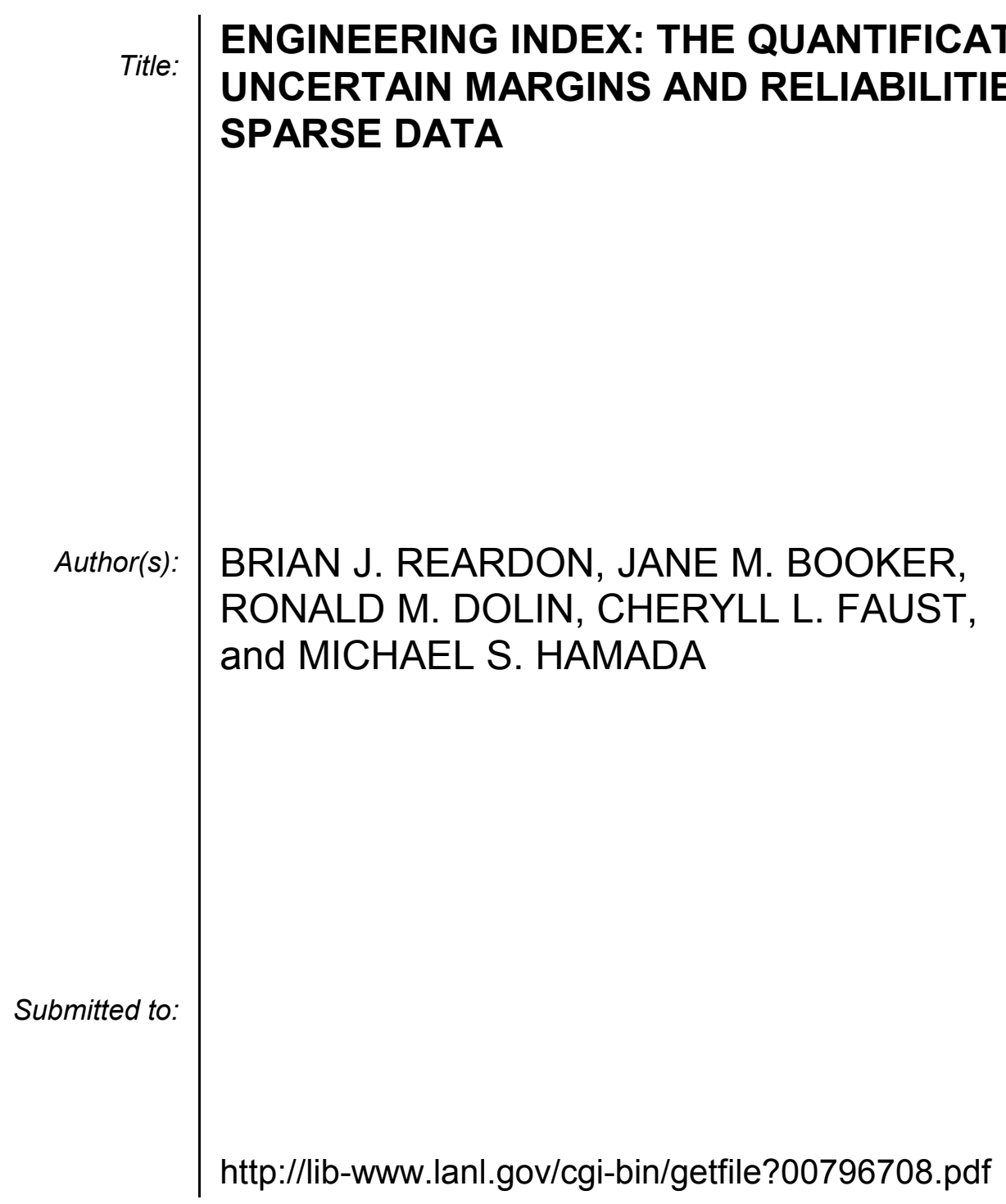

Los Alamos National Laboratory, an affirmative action/equal opportunity employer, is operated by the University of California for the U.S. Department of Energy under contract W-7405-ENG-36. By acceptance of this article, the publisher recognizes that the U.S. Government retains a nonexclusive, royaltyfree license to publish or reproduce the published form of this contribution, or to allow others to do so, for U.S. Government purposes. Los Alamos National Laboratory requests that the publisher identify this article as work performed under the auspices of the U.S. Department of Energy. Los Alamos National Laboratory strongly supports academic freedom and a researcher's right to publish; as an institution, however, the Laboratory does not endorse the viewpoint of a publication or guarantee its technical correctness. 


\title{
ENGINEERING INDEX: THE QUANTIFICATION OF UNCERTAIN MARGINS AND RELIABILITIES WITH SPARSE DATA
}

\author{
BRIAN J. REARDON, JANE M. BOOKER, RONALD M. \\ DOLIN, CHERYLL L. FAUST, MICHAEL S. HAMADA \\ Los Alamos National Laboratory
}

\begin{abstract}
The Engineering Index (EI) provides a measure of goodness for engineered systems, subsystems, components, and product functions. The EI supports certification and planning endeavors by assessing both a product's current state as well as inferring how a system potentially changes over time relative to their requirements. This work will show how Bayes Theorem can be used to accomplish this inference. The inference available through EI allows decision makers to plan for, and possibly mitigate, problems ahead of a crisis by estimating how a product's changes impacts system performance.
\end{abstract}

KEYWORDS: Uncertainty Quantification, Capability Index, Bayesian Inference

\section{INTRODUCTION}

Most engineered products are designed and manufactured with many levels of margin or factors of safety between the nominal requirement and the actual performance threshold. Figure 1 shows an example of the factors of safety implemented by different levels of engineers in the design and manufacturing process. Each engineering level is focused on producing parts that do not violate the safety limits imparted by the previous level. Since the engineers know that there is a degree of variation in all the parts they design or make, they tend to set conservative limits. The net result of such conservation is a very high reliability where reliability is defined as the probability that the product performs its intended function, which here means the percentage of parts that exceed the performance threshold. Additionally, this procedure also results in a large margin where margin is defined as the distance between the performance threshold and the worst performing product that still exceeds the threshold requirements. 


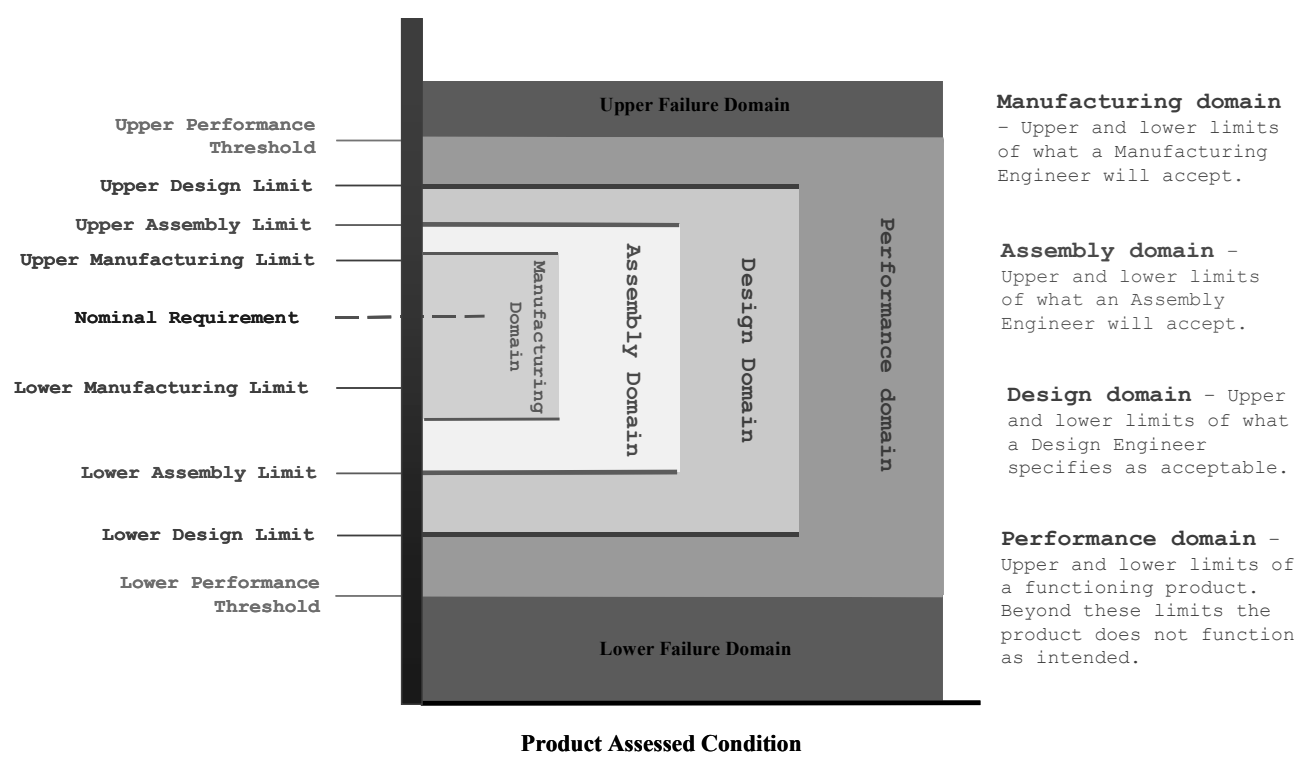

Figure 1. Illustration of the different levels of engineering limits placed around the nominal value.

A significant amount of product variation can still occur within the tight confines of the manufacturing limits. This variation or uncertainty arises from many potential sources. For example the inherit variability of the process, measurement uncertainty, and lack of knowledge. Generally speaking, this uncertainty is not considered detrimental so long as it stays well within the specified limits. In other words, uncertainty and variability are fine so long as they do not impact the reliability of the product.

A significant complication in the calculation of product reliability can occur, however, in situations where it is known that the performance of a product degrades with time. Most engineered products undergo some (albeit in many cases small) change in properties or dimension with time due to applied stress, radiation, corrosion, etc. This 'aging' is not really a problem for the engineer provided it is well known how the product ages and all the products are exactly the same. Unfortunately, these two ideal situations are rarely observed in practice. Thus, the engineer is left with the challenge of determining how the distribution of products changes with time and how these changes impact the reliability of the product. This is especially challenging given that the aging model of the product may be highly speculative. There may not be much dependable aging data available, and the errors associated with extrapolation and inference into the future may be large.

A further complication in the reliable and meaningful prediction of the performance of a product over time is the fundamental definition of reliability itself. As stated previously, reliability is defined as the percentage of the population that meets or exceeds the minimum performance threshold. Thus, the as built population of products may have a reliability of close to $100 \%$ initially. As the population ages the distribution may change significantly. The mean performance value may drift and the variance may widen. However, so long as all the products stay above the performance threshold, the reliability is unchanged. This fact presents an obvious problem with using reliability as a 
measure of the state of health of a system, since it does not properly characterize the population changing with time.

Consequently, a measure is needed that allows the engineer to estimate the state of health of a system before it takes a hit in reliability. The measure introduced here is similar to the capability index of the manufacturing arena ${ }^{1}$ and is referred to as the Engineering Index.

The Engineering Index (EI) was first proposed by J. Booker, R. Dolin, C. Faust, and M. Hamada as a means for assessing the goodness of an engineered system, subsystem, component, or function. The EI primarily measures the extent to which an engineered product is over-designed relative to its design/performance requirements. The quantification of a product's over-design is often encapsulated within the term "margin." A common metric in statistical process control is margin over uncertainty. This metric is referred to by names, including $\mathrm{C}_{\mathrm{pk}}{ }^{1}$ and Figure of Merit (FOM). It could loosely be described as the amount a product exceeds in design/performance requirements relative to the amount of uncertainty there was in assessing the margin.

Figure 2 shows how the Engineering Index is measured. The population of products has an initial accessed condition (IAC). The IAC could be the mean, median or any other measure of the distribution that is appropriate. The distribution also has a lower limit on the accessed condition $\left(\mathrm{AC}_{\text {low }}\right)$. The $\mathrm{EI}$ is then defined as $\left(\mathrm{AC}_{\text {low }}-\mathrm{PT}\right) /(\mathrm{IAC}-\mathrm{PT})$ where $\mathrm{PT}$ is the performance threshold. The elegance of the EI lays not only in its simplicity and intuitive nature but also in the fact that it changes with time even when reliability is a constant, thus giving the engineer an overall measure of the health of the system.

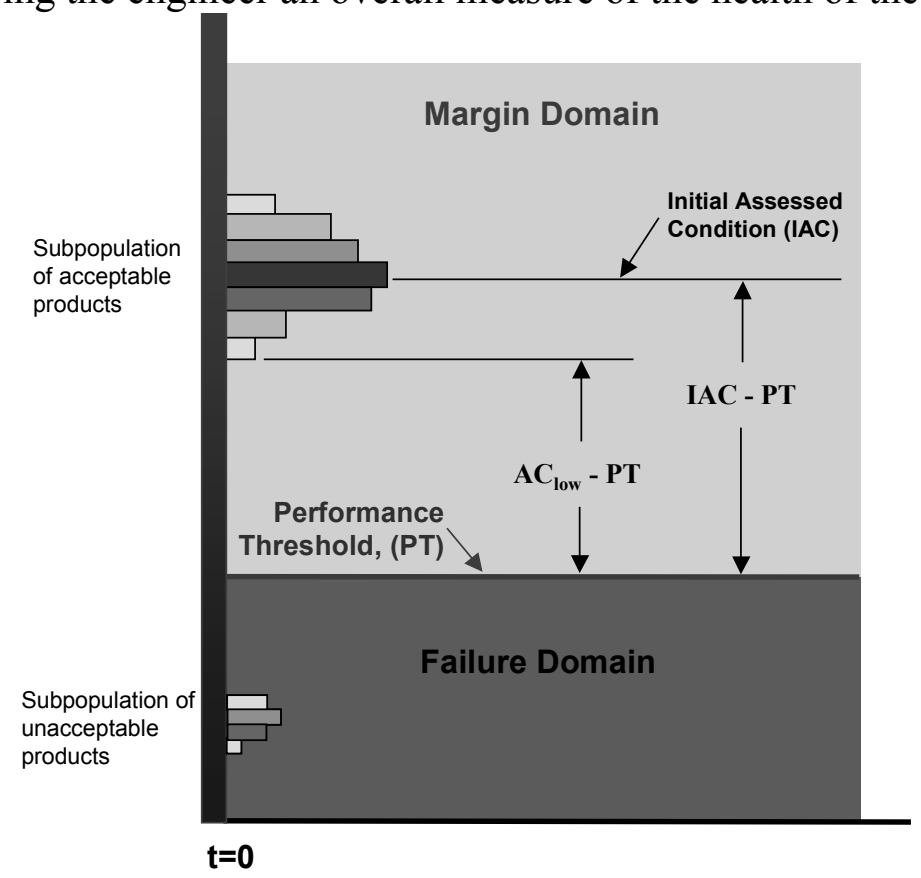

Figure 2. The definition of the terms in the engineering index $=\left(\mathrm{AC}_{\mathrm{low}}-\mathrm{PT}\right) /(\mathrm{IAC}-\mathrm{PT})$. 
Quantifying how the EI changes with time and how it can be accessed when little or no data is available is not trivial. This paper will highlight the use of Bayesian Inference to assist in the determination of the EI.

\section{PROCEDURE}

Figure 3 shows a possible product degradation scenario. Initially the subpopulation of acceptable products is assessed. If sample measurements are taken, then a sample distribution, such as the histogram shown, can be used and the lower assessed condition is defined as the minimum from any assessed condition to the performance threshold. In other words, the minimum margin condition. If analysis, or some synthesized combination, of analysis, measurements, and expert judgment are used to generate a continuous distribution, the lower assessed condition is found by truncating the tail region in some manner (e.g., the minimum value of a simulation or the first percentile).

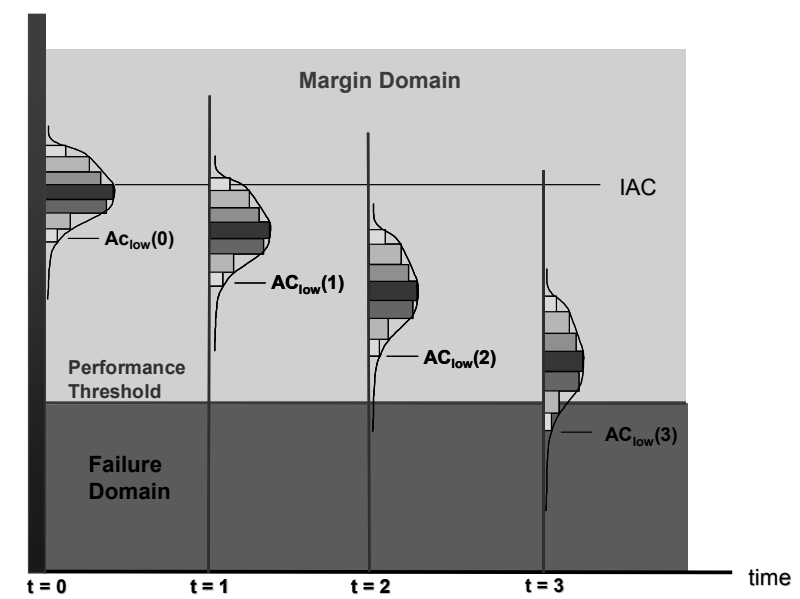

Figure 3: Possible product degradation scenario.

As a product ages it changes. Notice in the possible scenario shown in Figure 3 the distribution changes each time the population is assessed. Notice also that the lower assessed condition is moving toward the performance threshold, eventually crossing it. While margin is declining in the first three assessments $(\mathrm{t}=0,1$, and 2$)$, reliability remains constant because the area of the acceptable product distribution is constant even though the shape of the distribution is changing. By the fourth assessment $(t=3)$, there is no margin left in the product population and adjustments to the assessed reliability occur.

Figure 4 shows how this approach can be used to infer into the future. Once some knowledge is gained on how the subpopulation of acceptable products is declining over time, statistical inference techniques can be used to predict where in the future the EI(t) curve crosses the performance threshold. 


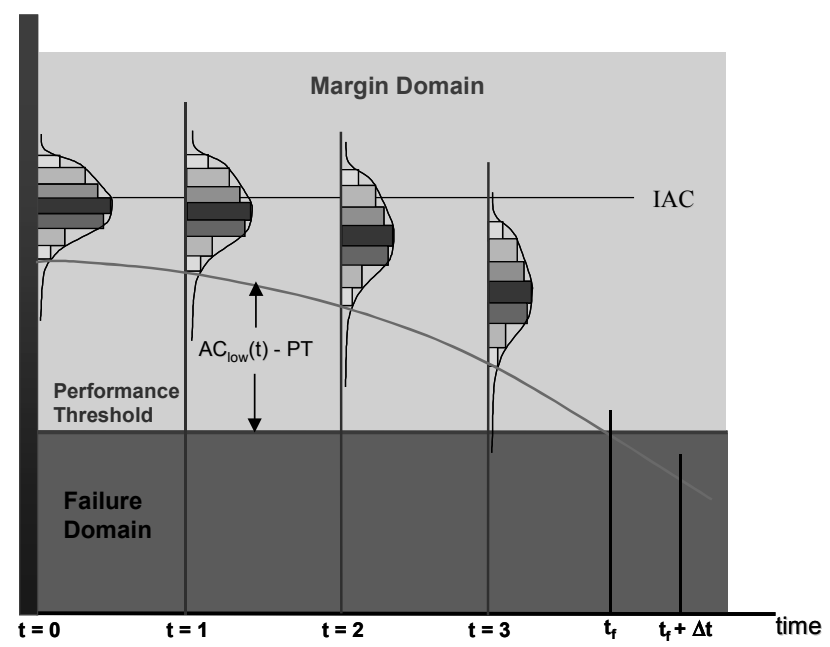

Figure 4: Inferring into the future.

Figure 5 shows a couple of additional features of an EI. Specifically, as an engineer infers into the future, past his last assessment, the uncertainty bounds upon an inferred assessed condition grow. The farther into the future an engineer assesses, the larger the uncertainty becomes. Quantifying this phenomenon can be a key to helping defend knowledge acquisition endeavors such as testing, or surveillance.

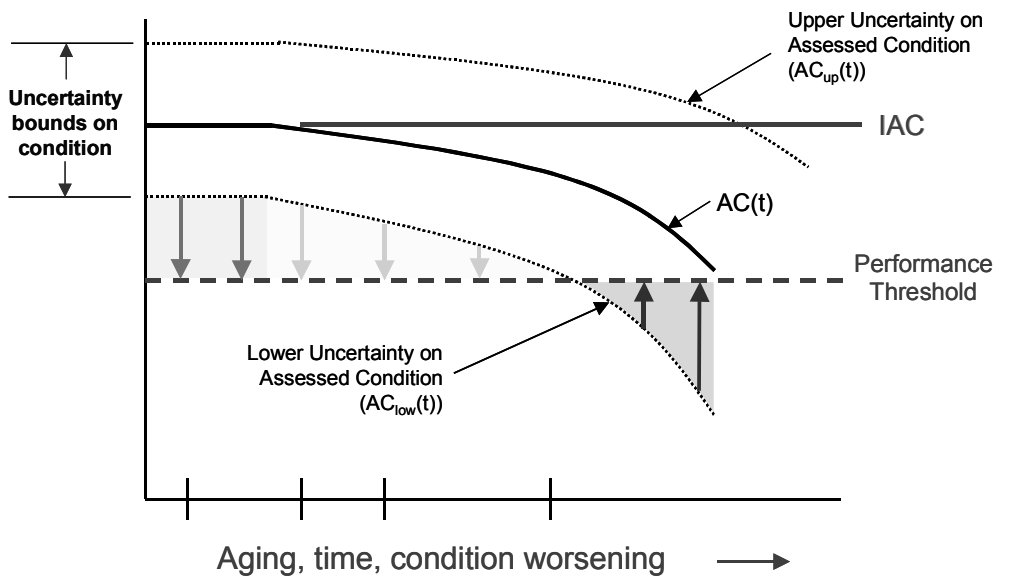

Figure 5. Impact of changes over inferred times.

Generally, one may have little or no data upon which to infer future behavior or even present state distributions. The most commonly used tool in such a situation is Bayesian Inference $^{2}$. The essence of Bayesian Inference rests on Bayes' Theorem: $p(\theta \mid y)=p(\theta) p(y \mid \theta) / p(y)$ which states that the probability of a model $(\theta)$ being true given the data $(y)$ (the posterior) equals the probability of selecting that particular model 
$p(\theta)$ (the prior) times the probability that the model will give the observed data $p(y \mid \theta)$ (the likelihood) normalized with the probability of observing $y, p(y)$. Where, $p(y)=\int_{\theta} p(\theta) p(y \mid \theta) d \theta$.

For the example discussed in Figures 4 and 5, assume that the data $(y)$ is being sampled from a normal distribution that changes with time: $y(t) \sim \mathrm{N}(\mu(t), \sigma(t))$ where, $\mu(t)=f\left(t, \theta_{\mu}\right)$ and $s(t)=f\left(t, \theta_{\sigma}\right)$. Further, $\theta_{\mu}$ and $\theta_{\sigma}$, are also sampled from normal distributions such that $\theta_{\mu} \sim \mathrm{N}\left(\mu_{\theta_{\mu}}, \sigma_{\theta \mu}\right)$ and $\theta_{\sigma} \sim \mathrm{N}\left(\mu_{\theta \sigma}, \sigma_{\theta \sigma}\right)$.

Assuming that the model, $\theta$, of Bayes Theorem above is a vector defined as $\left[\theta_{\mu}, \theta_{\sigma}\right]$, the prior, likelihood, normalization factor and finally the posterior are easily calculated for a particular datum, $y$. As new data becomes available the posterior from previous analysis becomes the prior of the new analysis.

Naturally, the discussion above is for illustrative purposes only. The numerical complexities of this problem are greatly simplified if it could be assumed that either the variance or mean of $\theta$ were constant. Another important and often overlooked point is that for most real world applications, normal distributions should not be assumed.

\section{CONCLUSIONS}

This work introduces the concept of an Engineering Index, which draws many parallels with capability indices. The Engineering index is a ratio of the initial accessed performance of a population to its margin from the performance threshold at time, $t$. This ratio changes as the population ages and thus gives a measure of how quickly the system is degrading even when the reliability of the system is constant.

Through the incorporation of a time dependent Engineering Index in conjunction with the formalisms of Bayesian Inference, we have shown, conceptually, the ease with which reasonable predictions can be made regarding the rate of degradation of a system. The main advantage of this approach rests in the fact that Bayesian Inference has proven quiet reliable in situations of sparse data and unclear performance requirements.

\section{ACKNOWLEDGEMENTS}

This work has been funded by the Department of Energy and the National Nuclear Security Agency.

\section{REFERENCES}

1. Kane, V. E., "Process Capability Indices," Journal of Quality Technology, Vol. 18, No. 1, 1986.

2. Gelman, A., Carlin, J.B., Stern, H.S., Rubin, D.B., Bayesian Data Analysis, Chapman \& Hall, 2000. 